\title{
To identify Zanjan food and beverage clusters
}

\author{
S. Hagh Nazari \\ Agriculture Faculty of Zanjan University, Iran
}

\begin{abstract}
Nowadays, industrial clusters have attracted the public attention in some parts of the world. The main power in the cluster is due to the creation of potential jobs, the same factor in the past to promote small industries, especially in the food and beverage field. In addition, the success of these clusters indicates the competitive ability of small and medium-sized companies in global markets. In this project, a new method was used called analysis of geographic information for identifying food and beverage factories in Zanjan Province. For this purpose, initially, SMEs were specified in Zanjan Province with 99 to 100 percent development and employment for 3 to 200 people in the mentioned field. So the fast and more accurate general perceptions resulted in the current status of industry-specified locations, how their distribution was carried out, the changes to economic factors over time, and future forecasts. In the next stage the geographic information of SMEs was obtained and analyzed. The nearest neighbour determination method was used to detect the acceptable scattering rate of industrial clusters. The results of the study showed that food and beverage cluster was in the third category of importance in the province after textile and electrical machinery clusters. So, in the future, based on the capabilities and guidance and support, the food and beverage clusters will grow.
\end{abstract}

Keywords: food clusters, geographic information, industrial clusters.

\section{Introduction}

In most industrialized countries, the importance of small industries has been accepted as a reality, increasing their share of industrial production and job creation, and increasing the approach to economic change and small units of production and employment. As a result of all these developments, small industries have more benefit than large industries. But in Iran small-scale industry only produces about $30 \%$ of the value of industrial production, although 
it provides an important contribution in terms of employment opportunities in the industrial sector $(56 \%)$.

Because of difficulties in supporting small and scattered units for the Iranian government and their heavy costs, it is necessary to organize them as industrial clusters. Considering the existence of embryonic and incomplete clusters in many areas of this country, it seems an attempt must be first directed to identify and secondly organize units in the form of the industrial clusters to set the growth and increase communications between them and lead to synergy (Shafiee [1]).

\section{Research methodology}

Researchers have used different qualitative and quantitative methods to determine the categories of industrial and commercial cluster units, for example, the UNIDO method for measuring various aspects of cognitive studies of clusters, the amount of input and output units tables (Input-Output), to determine value chain in the unit, using factor analysis, or share a place of employment Location Quotient, Methods and resources, etc.

\subsection{GIS analysis of clusters}

In this project, a new method called analysis of geographic information was used to identify industrial clusters. This method can be also used for planning in the provincial economic and industrial studies. Because of the accuracy and reliability of this tool, geographic information analysis was used in this project.

For this purpose, geographic information of three plans in Zanjan Province were used, they contained: 1) "the dispersion of province's schools information" 2) "an overview geographic information plan" done by Zanjan industry and mines organization 3) project being done "to determine the pollutant industries in the province "by environment organization in Zanjan Province. In this project the local place characteristics of small and medium sized units with 99 to 100 percent development and employment for between 3 and 200 people were used to determine small and medium industrial units.

In the next stage the geographic information was analyzed. The nearest neighbour determination method was used to detect acceptable scattering rate of industrial clusters. This method conducted first time by America's economic development department in the design of local clusters in 2006 (Fesser [2]).

In cluster analysis methods, the distance between nearest neighbours were considered by using the nearest neighbours, and how random distributions of clusters were determined. From a geographic point of view, closeness of the cluster units is also being used as the criteria for assessment of specialized clusters on their own. The average nearest neighbour index number differs between zero and above zero. If the variables were being smaller than this number, indicated potential for industrial and commercial clusters. For example, zero shows complete model units to be specified for clustering, while the number one expresses random distribution of units, and numbers higher than 2 indicate 
regular distribution units. This parameter must be interpreted with $\mathrm{Z}$ score. This means that the amount of points should not show so normal distribution units in the cluster distribution.

\subsection{Calculation of the nearest neighbour index or average nearest neighbour}

Index of the nearest neighbour is obtained by dividing the ratio of the observed distance on the average distance between neighbours in a hypothetical random distribution

\section{Results}

Identification of food and beverage industry clusters in Zanjan Province using geographic information analysis:

\subsection{Industrial cluster of food and beverage products in Zanjan Province}

Zanjan Province has various types of food technologies due to the high possibilities of diverse agricultural products. There are concentrated industrial units in Zanjan and Abhar Counties equal to 314 and 56 units respectively with $100 \%$ active development and employment numbers below 200 people. Tarom and Khodabandeh Counties are not accepted as clusters focus. Khoramdarreh County despite of having 22 units of food industries, cannot be accepted as a food cluster due to the diversity of units. Figure 1 shows the distribution map of industrial food and beverage products units in Zanjan Province.
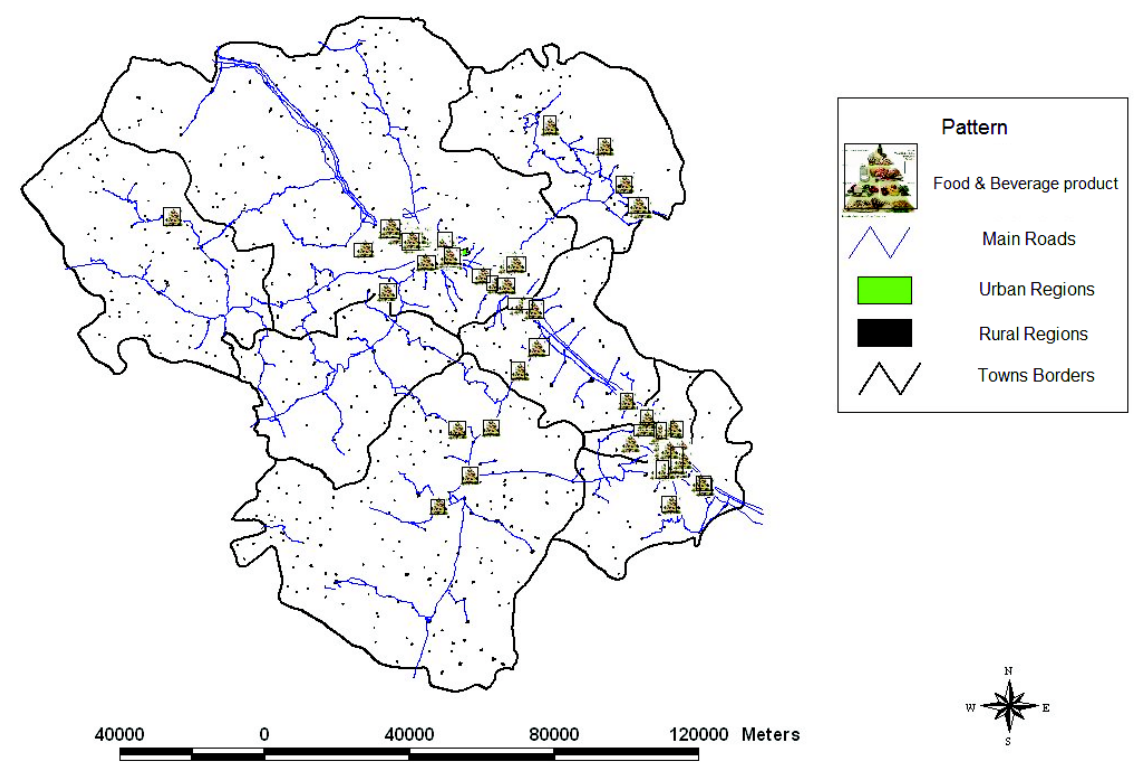

Figure 1: Distribution map of industrial food and beverage products in Zanjan Province. 
Geographic concentration of industrial food and beverage products was obtained in Zanjan Province after analysis by arc GIS software and shown in table 1. The nearest neighbour index in the province indicates that the potential for creating food clusters are suitable in the province.

Figure 2 shows detailed scattered industrial food and beverage products as a red box in the province. So, geographic concentration in the province for food industry units is significant with confidence $99 \%$ in some counties.

\section{Clustered}
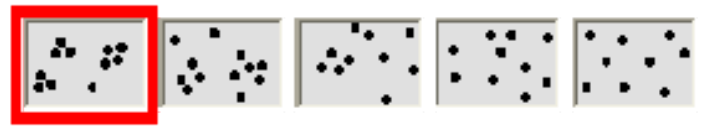

\section{Dispersed}

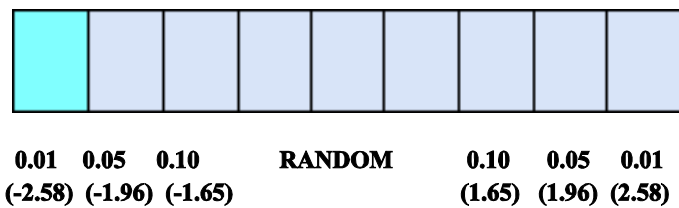

Significance Level:
Critical Values: $(-2.58)(-1.96)(-1.65)$

(1.65) (1.96) (2.58)

There is less than $1 \%$ likelihood that this clustered pattern could be the result of random chance.

Figure 2: Detailed scattered industrial food and beverage products in Zanjan Province. (See online for colour version.)

\subsection{Industrial cluster of food and beverage products in Zanjan County}

Food focus units can be seen in the central part of Zanjan city. Analysis of the nearest neighbour index and $\mathrm{Z}$ score in table 1 shows there are much better opportunities in food cluster units in Zanjan County rather than Zanjan Province. Because Nearest Neighbour Ratio value in the Zanjan County is smaller than this value in the province. The following features scattered cluster is shown in Zanjan County in a red outlined box:

\subsection{Industrial clusters of food and beverage in Abhar County}

So as mentioned earlier, multiplicity and concentration of food industry units in Abhar is in the second position in the province after Zanjan County. Also, via statistical analysis can also be realized that geographic focus of food units is in Abhar with 99\% significant confidence. Analysis of geographic concentration factors have been shown in table 1. Also, the detailed industrial units scattered food and beverage products in Abhar County shows there is less than $1 \%$ likelihood that this clustered pattern could be the result of random chance. 


\subsection{Industrial cluster of food and beverage products in Khorramdareh County}

There are about 22 units of food and beverage industries in Khorramdareh County, having an appropriate geographic focus of cluster in it, but a high diversity of food products, and will be inappropriate conditions for selection it for the cluster. Therefore it cannot be introduced as a cluster.

\subsection{Industrial food and beverage products in the Khodabandeh County}

Food units are few and very diverse and scattered in Khodabandeh County. Therefore this county is ineligible to vote to be an industrial cluster. Analysis of the nearest neighbour index and $\mathrm{Z}$ score in Khodabandeh County (table 1) reflecting the very poor status of the city being a possible cluster units of food. On the other hand active units are too few in this field, so it is not recommended now as the industrial cluster.

Distribution visual interpretation of this cluster is shown as a red outlined box in figure 3:
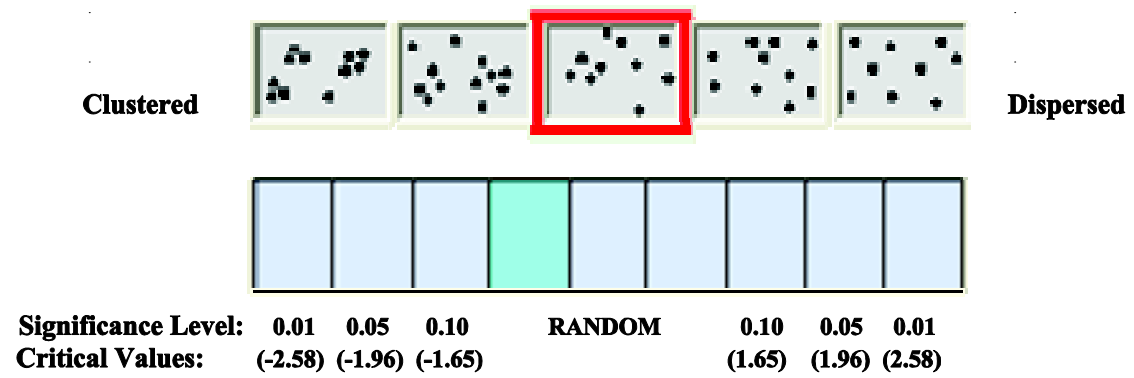

While somewhat clustered, the pattern may be due to random chance.

Figure 3: Detailed scattered industrial food products and beverages in Khodabandeh County. (See online for colour version.)

Analysis of the nearest neighbour index and $\mathrm{Z}$ score in Khodabandeh County (table 1) reflecting the very poor status of the city being a possible contender for cluster units of food. On the other hand active units are too few in this field, so it is not recommended now as the industrial cluster.

\subsection{Industrial cluster of food and beverage products in Tarom County}

There are 23 food industry units in the field of olive oil refinery and its packaging with 458 workers in Tarom County. Analysis of geographic factors and detailed scattered industrial food products and beverages in Tarom County shows while somewhat clustered, the pattern may be due to random chance.

Analysis of nearest neighbour index and Z score in Tarom County (table 1) is reflecting the very poor status of this county being possible for food industry 
cluster due to decentralization. On the other hand active units are too few and various in this field, so it is not recommended as the industrial cluster at present time.

Table 1: Comparison of quantitative geographic factors of industrial food and beverages clusters in Zanjan Province.

\begin{tabular}{|c|c|c|c|c|c|}
\hline $\begin{array}{l}\text { Cluster } \\
\text { Type }\end{array}$ & City & Z Score & $\begin{array}{l}\text { Nearest } \\
\text { Neighbour } \\
\text { Ratio }\end{array}$ & Expected & $\begin{array}{l}\text { Nearest } \\
\text { Neighbour } \\
\text { Obs. Mean } \\
\text { Distance }\end{array}$ \\
\hline & Zanjan & $\underline{17.82}$ & $\underline{0.146104}$ & $\underline{3768.831314}$ & $\underline{550.639627}$ \\
\hline Food & Abhar & $\underline{6.592514}$ & 0.360087 & 5079.680870 & 1829.125047 \\
\hline products & Khoramdareh & 3.287594 & 0.503914 & $\underline{2914.510228}$ & 1468.663408 \\
\hline and & Khodabandeh & 1.617238 & 0.577319 & 17938.255065 & 10356.087853 \\
\hline beverages & Tarom & 1.599670 & 0.658631 & 9398.162918 & 6189.923235 \\
\hline
\end{tabular}

\section{Discussion and conclusion}

The arrangements of clusters based on the sum of quantity geographical factors were obtained in Zanjan Province's Counties.

With surveying the above results in table 1, we can conclude that all counties of Zanjan Provinces do not have an equal opportunity for being a food industrial cluster. In the near future only Zanjan, Abhar, Khoramdareh in Zanjan Province, have a chance to make a successful food industrial cluster after reforming due to geographic and historical situation as Doeringer and Terkla [3] believe that geographical concentrations of industries gain performance advantages through co-location and cluster corporations.

Some empirical evidence offer interesting findings about industrial clusters in different counties of Zanjan Province, which are useful for the interpretation of this project's results. For example, in research were done by Bergman and Feser, on North Carolina clusters, researchers concluded that the higher focus on geographic clusters caused, faster development running in their size and greater coherence within the cluster (Fesser [2]).

As concluded from the theory and practice studying in Zanjan Province, its situation has caused industrial clusters creation in various shapes because of geography and social status capabilities in this area during the long years of its history, and its location in the way of the Silk Road, trading, industrial and strategic position which have led to a collection of traditional, industrial and commercial bases around the province. This is similar to that which Schmidt and Nadvy [4] expressed on how to boost industrial growth and competitive capabilities by making the process of clustering. They recorded that there are 
some clusters that have little mobility, while other clusters can increase their competitive ability and have found ways to international market. In formed clusters in developing countries, medium and large companies often play an important role in leading those clusters. Also, as Yamawaki [5] concluded that the emergence of Japan's clusters is related to historical circumstances, prior existence of large manufacturers, prior existence of supporting industries, prior existence of related industries in neighbouring geographic areas, reduction in transportation costs, regional government policy, and technology transfer.

Zanjan Province clusters' activities for automatic actions have been prevented mostly due to having some defects and limitations for full growth and maturity. Lack of communication between units and the fear of common business hazards have caused the halting of clusters' developments, so, making up the clusterspecific features available in them. Whereas according to Wallner research [6], ecology, economy and sociology, are important components of the complex industrial systems.

Zanjan Province's undeveloped clusters have incurred in the vicious circle of fragile competition, and yet abandoned in their positions. Therefore the profit and efficiency of their work is diminished day by day. So the main problem of these clusters is being isolated from society and modern economy and lack of active deal maker flexible support for coordination in order to eliminate defects in their cluster as Brown et al. [7] and Bell [8] concluded that the effects of foreign networks have great importance in developing clusters and helping small and medium enterprises for internationalization.

In the final review conducted by this research project over cluster in Zanjan Province, it was concluded that for the development of all clusters we cannot use the same pre-determined plan. Te development plan for each cluster is determined based on the needs dictated by the stakeholders in each period. Once this approach to cluster development is faced with the demand in a wide range of sectors, it is necessary to spread an intervention mechanism for preparing a flexible custom program development with good practice. Also, Zhu and Ding [9] suggested the new idea of reconstruction of industrial location as to cultivate regional innovative system.

In general, to correct the defects of Zanjan Province clusters, the following actions should be carried out primarily and jointly:

- Strengthen links within the cluster, including communications between units within the cluster, and between these units and support agencies, governments, banks, business development centers and more.

- Coordinate activities, in the direction of sharing the whole cluster's interests

- Leading the cluster to independence and self sufficiency by a cluster development's agent or CDA

- Building confidence to cluster developing process by cluster development factors in units located in clusters. 
As the idea of Chiaroni and Chiesa [10], the key features which currently play a key role in a cluster success are: (i) formal input-output relationships, (ii) buyer-seller linkages, (iii) geographic concentration of firms, and (iv) shared specialized infrastructures.

\section{Conclusion}

Theory and practical reviews in this project showed that within small and medium industries in Zanjan Province, food and beverage clusters have the aptitude for completing and development after the textile and electrical machinery clusters. So, in the future, food and beverage clusters will grow based on the capabilities and guidance and support.

\section{Suggestions and strategies}

For reforming of food and beverage clusters bellow suggestions and strategies can be considered in Zanjan Province:

- Review and determine the weaknesses points of industry clusters based on the type of food related products and try to resolve them.

- Government support for actively participate of professionals in order to consider the features of Iranian products in international and valid standards.

- To upgraded Technology in planting, harvesting and production efficiency and quality of agricultural products.

- To succeed in global competition, food production units must be supported by government in the fields of international trade.

- Research and laboratory units must be used to increase production efficiency in terms of quality and quantity for each cluster of specialty food industry under discipline.

\section{Acknowledgement}

The author gratefully acknowledges the very helpful comments and suggestions given by experts of industry and mine organization in the field of food section.

\section{References}

[1] Shafiee, section program planning and organizational development of small industries http://techstudies.org/ipolicy/printtext.php?adr=archive/82/8204/ S8204094.htm\&mode $=$ basic \&arr $=0 \&$ sha $=0 \&$ noe $=0 \&$ sub

[2] Feser E.J, Introduction to regional industry cluster analysis, Chapel Hill, North Carolina 2006.

[3] Doeringer P.B, Terkla D.G, Business strategy and cross-industry clusters. Econ Dev Q. 9, pp. 225-237. 1995. 
[4] Schmitz H, \& Nadvi K, Industrial clusters in less developed countries: Review of experiences and research agendas. Brighton: IDS. 1994.

[5] Yamawaki H, the Evolution and Structure of Industrial Clusters in Japan. Small Business Economics. 18: pp. 121-140. 2002.

[6] Wallner H.P, Towards sustainable development of industry: networking, complexity and eco-clusters. Journal of Cleaner Production 7. pp. 49-58. 1999.

[7] Brown, J.S., Duguid, P. Mysteries of the region: knowledge dynamics in Silicon Valley. In: Lee, C., Miller, W.G., Hancock, M.G., Rowen, H.S. Eds.), The Silicon Valley Edge: a Habitat for Innovation and Entrepreneurship. Stanford University Press, Stanford, CA, pp. 16-39. 2000.

[8] Bell, G.G., Clusters, networks, and firm innovativeness. Strategic Management Journal 26, pp. 287-295. 2005.

[9] Zhu H., Ding S., Reconstruction of Industrial Location in View of Industrial Agglomeration. Chinese Geographical Science 16.4. pp. 294298. 2006.

[10] Chiaroni D, Chiesa V, Forms of creation of industrial clusters in biotechnology: Technovation 26 pp. 1064-1076. 2006. 